\title{
A Sonographic Quantitative Cutoff Value of Cerebral Venous Outflow in Neurologic Diseases: A Blinded Study of 115 Subjects
}

\author{
L. Monti, E. Menci, P. Piu, S. Leonini, U. Arrigucci, M. Bellini, A. Zandonella, P. Galluzzi, and A. Casasco
}

\begin{abstract}
BACKGROUND AND PURPOSE: The autonomic nervous system maintains constant cerebral venous blood outflow in changing positions. Alterations in cerebral autoregulation can be revealed by postural changes at quantitative color Doppler sonography. The aim of this study was to reach an optimal cutoff value of the difference between the cerebral venous blood outflow in the supine and seated positions that can discriminate healthy controls from patients with multiple sclerosis and those with other neurologic diseases and to evaluate its specificity, sensitivity, and diagnostic accuracy.
\end{abstract}

MATERIALS AND METHODS: One hundred fifteen subjects ( 54 with MS, 31 healthy controls, 30 with other neurologic diseases) underwent a blinded quantitative color Doppler sonography evaluation of cerebral venous blood outflow in the supine and sitting positions. An optimal difference value between the supine and sitting positions of the cerebral venous blood outflow cutoff value was sought.

RESULTS: The difference value between supine and sitting positions of the cerebral venous blood outflow was $\leq 503.24$ in 38/54 (70.37\%) patients with MS, $9 / 31$ (29.03\%) healthy controls, and 13/30 (43.33\%) subjects with other neurological diseases. A difference value between supine and sitting positions of the cerebral venous blood outflow at a 503.24 cutoff reached a sensitivity at $70.37 \%$, a $70.96 \%$ specificity, a $80.85 \%$ positive predictive value, and a $57.89 \%$ negative predictive value; the quantitative color Doppler sonography parameters yielded significant differences. The difference value between supine and sitting positions of cerebral venous blood outflow $\leq 503.24$ assessed the significant difference between MS versus other neurological diseases.

CONCLUSIONS: Alteration of cerebral venous blood outflow discriminated MS versus other neurologic diseases and MS versus healthy controls. The difference value between supine and sitting positions of cerebral venous blood outflow $\leq 503.24$ was statistically associated with MS.

ABBREVIATIONS: $\mathrm{AUC}=$ area under the curve; $\mathrm{CVF}=$ cerebral venous blood outflow; $\Delta \mathrm{CVF}=$ difference value between supine and sitting positions of the cerebral venous blood outflow; $\mathrm{HC}=$ healthy controls; $\mathrm{OND}=$ other neurologic diseases

C omplete evaluation of the cerebral venous circulation is difficult due to its anatomic variability. In vivo study of this system began in the 1970 s by venography. ${ }^{1}$ Venography is still considered the criterion standard; however, only color Doppler sonography can evaluate dynamic aspects, including the efficiency of the jugular valves or flow characteristics in sitting and

Received August 24, 2013; accepted after revision November 26.

From the Unit of Neuroimaging and Neurointervention (L.M., E.M., S.L., U.A., M.B., A.Z., P.G.), Department of Neurological and Sensorial Sciences, Azienda Ospedaliera Universitaria Senese, Santa Maria alle Scotte General Hospital, Siena, Italy; Department of Medicine, Surgery and Neuroscience (P.P.), University of Siena, Siena, Italy; and Unit of Endovascular and Percutaneous Therapy (A.C.), Clinica Nuestra Señora del Rosario, Madrid, Spain.

Please address correspondence to Lucia Monti, MD, Unit of Neuroimaging and Neurointervention, Department of Neurological and Sensorineural Sciences,

Azienda Ospedaliera Universitaria Senese, "Santa Maria alle Scotte" General Hospital, Viale Bracci, 2-53100 Siena, Italy; e-mail: I.monti@ao-siena.toscana.it

http://dx.doi.org/10.3174/ajnr.A3864 supine positions. MR venography can be a noninvasive imaging technique for the morphologic detection of extracranial venous anomalies in the internal jugular and vertebral veins in patients with multiple sclerosis, but it cannot give a dynamic evaluation. ${ }^{2}$ Phase-contrast MR imaging was used to measure venous flow in the internal jugular and epidural veins but only in the supine position. ${ }^{3}$ MR perfusion demonstrates a hypoperfusion of white and gray matter, and the parameters involved are cerebral blood volume, cerebral blood flow, and mean transit time, but not cerebral venous blood outflow (CVF). ${ }^{4,5}$

Disorders involving the cerebral venous system may result in CVF insufficiency, elevation of venous pressure, and an increase of intracranial pressure and may lead to parenchymal abnormalities. Compliance of the venous system depends on anatomic variants and the onset timing of venous pathologies. Multiple sclerosis is defined as an inflammatory demyelinating disease of the CNS, with presumed autoimmune etiology, which occurs in ge- 

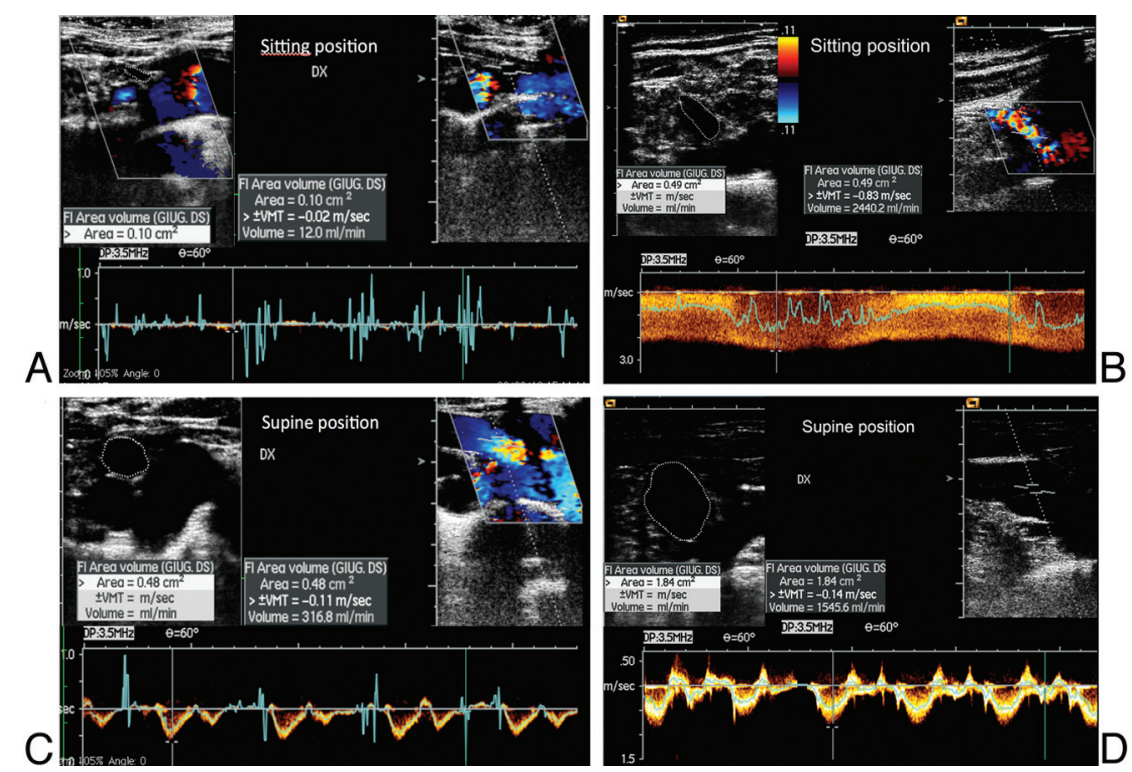

FIG 1. Quantitative evaluation of CVF in the supine and sitting positions in $\mathrm{HC}(A$ and $B)$ and patients with MS ( $C$ and $D)$. The $\Delta C V F$ was $>503.24$ in $\mathrm{HC}$ and $<503.24$ in patients with MS.
The sample included 115 consecutive subjects ( 81 women and 34 men; mean age, $42.25 \pm 11.2526$ years), including 54 (43 women and 11 men; mean age, $42.24 \pm 9.66$ years) patients with MS, 31 (19 women and 12 men; mean age, $36.64 \pm 9.46$ years) age-matched healthy controls, and 30 (19 women and 11 men; mean age, $48.93 \pm 12.11$ years) patients with OND, including patients with different defined neurologic diseases with autoimmune etiology, such as cerebral vasculitis $(n=16)$, neurosarcoidosis $(n=2)$, or chronic cerebral venous sinus thrombosis $(n=2)$; Parkinson disease $(n=4)$; and epilepsy $(n=6)$. The recruitment of those with nonoverlapping pathologies could test whether $\triangle \mathrm{CVF}$ is strongly correlated to patients with MS.

Patients with MS were divided into 2 subgroups (ie, subgroup 1, including 40 with relapsing-remitting MS, and netically susceptible individuals. Recently, a causal relation between the cerebral venous system and MS has been suggested. ${ }^{6-8}$ Accordingly, the term "chronic cerebrospinal venous insufficiency" has been coined to identify a chronic state of impaired venous drainage from the CNS as a putative causative factor responsible for MS. Stenosis of the internal jugular veins and intra- and extracranial reflux have been suggested as a cause of this impaired outflow. The hypothesis is that venous reflux may lead to the accumulation of iron in the CNS, triggering autoimmune events. ${ }^{9,10}$

Although chronic cerebrospinal venous insufficiency in MS has not been supported by recent studies, ${ }^{11-14}$ it has forced research on possible vascular impairment in this complex multifactorial disease, including ischemic strokes, cerebral hypoperfusion, and venous blood drainage. ${ }^{15,16}$ In the literature, there are controversial results on chronic cerebrospinal venous insufficiency. Notably, a phenomenon such as cerebral venous impairment can be studied by evaluating other sonographic parameters or factors. Thus, the difference value between the CVF in the supine position and the seated position $(\Delta \mathrm{CVF})$ has been proposed and evaluated in a previous scientific article, ${ }^{17}$ in which MS and healthy controls (HC) groups were compared with a cutoff value of $\Delta \mathrm{CVF}=0$. With that decision threshold, $\Delta C V F$ findings were mainly negative in patients with MS, an opposite result to that in healthy subjects. ${ }^{18-20}$ A negative $\triangle \mathrm{CVF}$ is consistent with a reduced venous outflow in the supine position, resulting from a reduced venous system compliance in patients with MS.

The aim of the present study was to identify the cutoff value of $\triangle \mathrm{CVF}$ that maximizes the diagnostic accuracy of the model. Its specificity, sensitivity, and diagnostic accuracy in 3 different groups of patients, those with MS, those with other neurologic diseases (OND), and healthy controls, were evaluated.

\section{MATERIALS AND METHODS}

This study was approved by the Ethics Committee of our institution, and written informed consent was obtained from all subjects.

1382 Monti Jul 2014 www.ajnr.org subgroup 2, including 14 with primary- and secondary-progressive MS ( $n=1$ and $n=13$, respectively). No patients with clinically isolated syndromes were admitted; therefore, none were enrolled.

All patients underwent neurologic assessment before quantitative color Doppler sonography examination. The degree of disability was assessed by using the Expanded Disability Status Scale; arm/hand dexterity was tested by Nine Hole Peg Test; and leg function, by the timed 8-Meter Walk Test.

Quantitative color Doppler sonography was performed by 2 skilled neuroradiologists (E.M. and L.M.) with experience in the sonography field who were blinded to the patient history and clinical status.

A color-coded sonography system (Sequoia; Siemens, Erlangen, Germany), a 7- to 9-MHz linear probe, and a 2.5-MHz sector probe were used. The interobserver concordance was evaluated by the examination of 30 randomly selected subjects (ie, 10 subjects from each of the 3 groups) who had been examined separately by the 2 neuroradiologists, each one blinded to the results obtained by the other. Discrepancies were resolved through discussion to produce consensus assessments.

The $\Delta C V F$ was evaluated in all the subjects. The outflow of the internal jugular and vertebral veins was calculated from the time average velocity (TAV) and the cross-sectional area (CSA) of the vessel $(\mathrm{CVF}=\mathrm{CSA} \times \mathrm{TAV})$. The time average velocity was measured during a minimum of 3 cardiac cycles at the end of the expiratory phase. ${ }^{21-23}$ The CVF of each vein was calculated in both clinostatism and the seated position. The sum of all the venous flows was then calculated in clinostatism and the seated position (Fig 1). The difference between the clinostatism and seated position is the $\Delta$ CVF value. ${ }^{17}$

A positive cutoff value of the $\Delta$ CVF between the different groups was sought, as well as its sensitivity, specificity, and diagnostic accuracy. The relationship among the values of $\triangle \mathrm{CVF}$ and age, sex, and clinical status was considered. 


\section{Statistical Analysis}

The reliability of the results obtained by 2 operators was calculated by using the Fleiss $\kappa$ index. The frequency distributions of the $\Delta$ CVF cutoff value among the subjects in the MS, HC, and OND groups were displayed as contingency tables. The differences between the proportions of the outcomes of this diagnostic index over the MS, HC, and OND groups were assessed through the Marascuilo procedure, which enabled simultaneous testing of the differences of all pairs of proportions. The Kruskal-Wallis test was applied to compare the distributions of the $\Delta C V F$ cutoff value among the groups and to evaluate the differences among the subclasses of MS disease. In either case, the post hoc tests were performed by the Dunn multiple comparison test.

All the statistical tests were 2-tailed, and the significance level was fixed at .05.

The errors of classification were reported in terms of sensitivity, specificity, negative predictive value, and positive predictive value (PPV), along with their $95 \%$ confidence intervals. The odds ratio was also provided, and its $P$ value was determined by the Fisher exact test. The capability of the $\Delta$ CVF cutoff value to classify the MS forms (relapsing-versus-progressive) was reported as ORs.

The cutoff (ie, the threshold of the $\Delta \mathrm{CVF}$ ) had been initially set to zero-namely the negative values of $\Delta C V F$ were considered prognostic of pathologic status or "events," while the positive values of $\Delta \mathrm{CVF}$, as predictive "nonevents." By increasing the level of the threshold, we expected to decrease the number of the false-negative predicted cases because $68.52 \%$ of the patients with MS had a positive $\Delta \mathrm{CVF}$.

Performances of the models were assessed by the receiver operating characteristic analysis curve, which is reported to be the most opportune approach and a comprehensive description and measurement of diagnostic accuracy because it estimates all of the combinations of sensitivity and specificity that a diagnostic test can produce. $^{24,25}$ The range of the cutoff values from which selecting the optimal threshold was formed by the percentiles of the distribution of the $\triangle \mathrm{CVF}$ in the HC group not only because the healthy condition is usually adopted as the reference standard in a diagnostic test, but also because the $\triangle \mathrm{CVF}$ distributions of $\mathrm{HC}$ and OND groups were largely overlapping. Every percentile was, in turn, set as the "potential best threshold" (this implies, from time to time, establishing, a priori, the specificity of the test). Then, in correspondence with each percentile, the number of subjects (from MS, HC, OND) with a $\triangle$ CVF lower than the potential cutoff was counted as an "event" (ie, abnormalthis means, from time to time, determining the sensitivity of the test). Hence, by varying the percentile, it has been possible to trace the relationship between sensitivity and specificity to give rise to the receiver operating characteristic analysis curve.

The optimal positive cutoff threshold was determined in correspondence to the best compromise among sensitivity, specificity, and PPV.

We measured the area under the receiver operating characteristic analysis curve (AUC); and its statistical significance against the null hypothesis of AUC $=0.5$ was assessed by means of the $Z$-test. ${ }^{26}$ The area under the curve can take values between 0.5 and 1.0. The greater the area under the curve (ie, the more the curve approaches the vertex of the graph), the greater the discriminating power of the test will be. For the interpretation of the values of the area below the receiver operating characteristic analysis curve, we referred to the classification proposed by Swets ${ }^{27}$ : AUC $=0.5$, the test is not informative; $0.5<\mathrm{AUC} \leq 0.7$, the test is slightly accurate; $0.7<\mathrm{AUC} \leq 0.9$, the test is fairly accurate; $0.9<\mathrm{AUC}<1.0$, the test is highly accurate; and AUC $=1$ is a perfect test.

The robustness of the $\Delta$ CVF model was tested by using by an independent ("test") sample made of 52 subjects with MS and 27 HC. Thus, the AUC of the test set was evaluated, and in correspondence to the best threshold estimated from the "training" set (ie, the given sample), we traced the values of sensitivity, specificity, and accuracy for the test set.

An internal test set (ie, a cross-validation test) is used for getting an independent OND sample by iterating the leave- $n$-out algorithm 2000 times. A different subset of the data (10 records) was held out each time, so that the training sets included 20 subjects and the outof-sample, 10 subjects. The medians of the classification errors obtained from each partition were calculated; then, the sensitivity, specificity, and diagnostic accuracy were assessed. Last, the AUCs measured from the training and testing samples were compared.

Logistic regression was applied to predict the realization of the variable $\Delta C V F$ dichotomized (according to the cutoff value), as a function of the demographic and clinical regressors-namely, age, sex and Expanded Disability Status Scale.

\section{RESULTS}

The Fleiss $\kappa$ index, calculated on 30 subjects ( 10 with MS, 10 HC, 10 with OND), was 0.9333 , and its confidence interval (95\%) was $0.8402-1.0264$. Therefore, the observed agreement between the 2 operators was not accidental $(z=5.1117, P<.0001)$.

An optimal cutoff value of the $\triangle C V F$ was reached at the 30 th percentile (ie, $\Delta \mathrm{CVF}=503.24$ ) of the $\mathrm{HC}$ data distribution. $\Delta \mathrm{CVF}<503.24$ was present in $38 / 54(70.37 \%)$ patients with MS, 9/31 (29.03\%) HC, and 13/30 (43.33\%) subjects with OND. The null hypothesis of equal proportions was rejected $\left(\chi^{2}=14.7584\right.$, $P=.0006$, power $=0.9405$ ).

By comparing MS versus $\mathrm{HC}$ groups with a cutoff of $\Delta \mathrm{CVF}=$ 503.24, the sensitivity was $70.37 \%$; the specificity, $70.97 \%$; the $\mathrm{PPV}, 80.85 \%$; and the negative predictive value, $57.89 \%$; the OR calculated for $\Delta \mathrm{CVF}<503.24$ was significant $(5.81, P=.00016)$. Given OND versus $\mathrm{HC}$, the sensitivity was $45 \%$; the specificity, $70.97 \%$; the PPV, 50\%; the negative predictive value, $66.67 \%$; and the $\mathrm{OR}$ was not significant $(\mathrm{OR}=2, P=.1091)$. If one compared MS and OND, the sensitivity was $70.37 \%$; the specificity, $55 \%$; the $\mathrm{PPV}, 80.85 \%$; the negative predictive value, $40.74 \%$; and the OR was significant $(2.90, P=.0103)$ (Table 1$)$.

The Kruskal-Wallis test allowed rejecting the null hypothesis that the observed $\triangle \mathrm{CVF}$ in subjects with MS, OND, and HC originated from the same distribution $(P=.0003)$. The post hoc test indicated the significant difference $(P<.01)$ between patients with MS and HC and between subjects with MS and OND. HC versus subjects with OND was not statistically different (Fig 2).

The Kruskal-Wallis test applied to compare HC and MS subgroups (relapsing-remitting, primary-progressive, and secondary-progressive) indicated a significant difference $(P=.0014)$, which was determined by relapsing-remitting versus $\mathrm{HC}(P<$ $.01)$ and primary-progressive/secondary-progressive versus $\mathrm{HC}$ $(P<.05)$. No statistically significant difference was assessed between the relapsing and progressive forms (Fig 3 ). 
Table 1: Analysis of classification errors: training sets ${ }^{a}$

\begin{tabular}{lccc}
\hline & MS vs HC & MS vs OND & OND vs HC \\
\hline Sen \% & 70.37 & 70.37 & 45 \\
$95 \% \mathrm{Cl}$ (Sen) & $58.19-82.55$ & $58.19-82.55$ & $23.20-66.80$ \\
Spe \% & 70.97 & 55 & 70.97 \\
$95 \% \mathrm{Cl}(\mathrm{Spe})$ & $54.99-86.95$ & $33.20-76.80$ & $54.99-86.95$ \\
$\mathrm{FP} \%$ & 29.03 & 45 & 29.03 \\
$95 \% \mathrm{Cl}(\mathrm{FP})$ & $13.03-45.01$ & $23.20-66.80$ & $13.05-45.01$ \\
$\mathrm{FN} \%$ & 29.63 & 29.63 & 55 \\
$95 \% \mathrm{Cl}(\mathrm{FN})$ & $17.45-41.81$ & $17.45-41.81$ & $33.20-76.80$ \\
$\mathrm{PPV} \%$ & 80.85 & 80.85 & 50 \\
$95 \% \mathrm{Cl}(\mathrm{PPV})$ & $69.60-92.10$ & $69.60-92.10$ & $26.90-73.10$ \\
$\mathrm{NPV} \%$ & 57.89 & 40.74 & 67 \\
$95 \% \mathrm{Cl}(\mathrm{NPV})$ & $42.20-73.59$ & $22.21-59.27$ & $50.58-82.75$ \\
OR & 5.81 & 2.90 & 2 \\
$95 \% \mathrm{Cl}(\mathrm{OR})$ & $2.20-15.33$ & $1.01-8.34$ & $0.62-6.47$ \\
\hline
\end{tabular}

Note-Sen indicates sensitivity; Spe, specificity; FP, false-positive; FN, false-negative; NPV, negative predictive value; PPV, positive predictive value.

${ }^{\text {a }}$ The columns refer to each comparison between the observed (ie, training) groups.

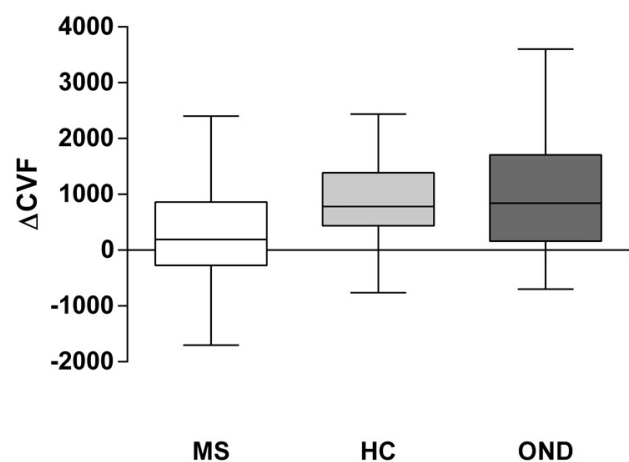

Kruskal-Wallis test

$P$ value $\quad 0.0003$

Kruskal-Wallis statistic $\quad 16.05$

\begin{tabular}{lllll}
\hline Dunn's multiple comparisons test & Mean rank diff. & Significant? & Summary & Adjusted P Value \\
MS vs. HC & -25.74 & Yes & $* *$ & 0.0018 \\
MS vs. OND & -24.09 & Yes & $* *$ & 0.0045 \\
HC vs. OND & 1.649 & No & ns & $>0.9999$
\end{tabular}

FIG 2. The $\triangle C V F$ distribution among the 3 groups. If one applies the Kruskal-Wallis test, significant differences result between MS and $\mathrm{HC}$ and MS and OND, while HC versus OND is not statistically different.

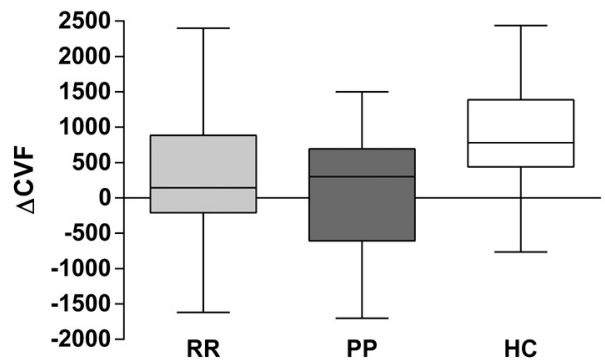

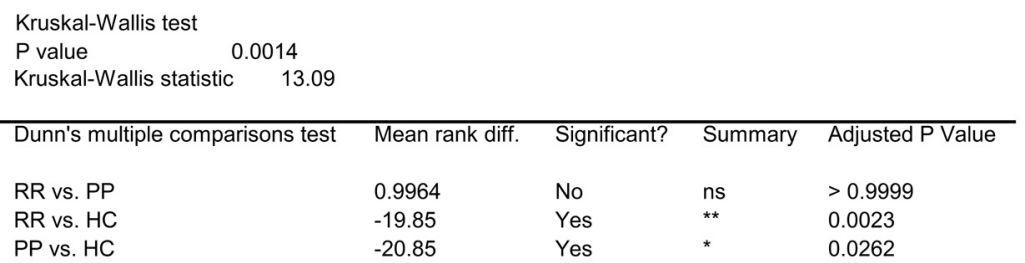

FIG 3. Boxplot $\triangle C V F$ and different subgroups of patients with $M S$. The Kruskal-Wallis test shows no significant difference among MS subgroups.
All the AUCs were different from one another-that is, the AUC was 0.7034 (standard error $=0.0564, P=.00015$ ) in the comparison between MS and HC (ie, fairly accurate), 0.7306 (standard error = $0.0597, P=.00001$ ) if the MS group was compared with OND (ie, fairly accurate), and 0.6323 (standard error $=0.0611, P=.0152$ ) when comparing OND versus $\mathrm{HC}$ (ie, slightly accurate).

In the independent sample, $\Delta \mathrm{CVF}<503.24$ was present in 41/52 patients with MS, 11/27 HC, and 4/10 subjects with OND (Table 2). Performance of the $\Delta \mathrm{CVF}<503.24$ model was also assessed on the independent sample (test set) by the analysis of the receiver operating characteristic analysis curve. The AUC was 0.7877 (standard error $=0.0505, z=5.7018, P=.00015$ ) in the comparison between MS and HC; the AUC was 0.8260 (standard error $=0.0591, z=5.5162, P=0)$ if the MS group was compared with OND; and the AUC was 0.55 (standard error $=0.1092, z=$ $0.4577, P=.3236)$ when comparing OND versus $\mathrm{HC}$. There was significant difference in the AUC values for MS versus $\mathrm{HC}(z=9.7015, P=0)$, MS versus OND $(z=-9.1021, P=0)$, and HC versus $\mathrm{OND}(z=-3.7631, P=$ .000083). The accuracy of the model was fair for the comparison between MS and $\mathrm{HC}$ and MS and OND, while it was not informative between OND and HC.

The criterion $\Delta \mathrm{CVF}<503.24$ applied within the MS subgroups to assess their capability to classify relapsing forms versus progressive forms resulted in 29/40 for relapsing-remitting and 10/14 for primary-progressive and secondaryprogressive, with $\mathrm{OR}=1.0545$, not significantly different from $1(P=.2674)$.

The logistic regression was applied to predict the realization of the variable $\triangle \mathrm{CVF}$ dichotomized according to the cutoff value, as a function of the demographic (age and sex) and clinical (Expanded Disability Status Scale; EDSS) regressors.

The implementation of the logistic model on the MS, HC, and OND groups did not result in the identification of significant effects of age, sex, and clinical status over the outcomes of $\triangle \mathrm{CVF}$. The $P$ values corresponding to these considered variables for MS, HC, and OND were respectively: $\left(P_{\text {age }}=.81 ; P_{\text {sex }}=.79 ; P_{\mathrm{EDSS}}=\right.$ $.75),\left(P_{\text {age }}=.77 ; P_{\text {sex }}=0.56\right)$, and $\left(P_{\text {age }}=\right.$ $\left..86 ; P_{\mathrm{sex}}=.82\right)$.

\section{DISCUSSION}

The cerebral venous system has very variable anatomic patterns, ${ }^{28-31}$ to maintain an efficient and normal CVF. Qualitative (ie, jugular valves or flow characteristics) and quantitative (ie, flow rate and velocity) aspects of CVF are demonstrated by using quantitative 
Table 2: Analysis of classification errors: out-of-sample sets ${ }^{a}$

\begin{tabular}{lccc}
\hline & MS vs HC & MS vs OND & OND vs HC \\
\hline Sen \% & 78.85 & 91.11 & 40 \\
$95 \% \mathrm{Cl}$ (Sen) & $67.75-89.95$ & $82.80-99.43$ & $9.64-70.36$ \\
Spe \% & 59.26 & 35.29 & 59.26 \\
$95 \% \mathrm{Cl}$ (Spe) & $40.73-77.79$ & $12.58-58.01$ & $40.73-77.79$ \\
FP \% & 40.74 & 64.71 & 40.74 \\
$95 \% \mathrm{Cl}(\mathrm{FP})$ & $22.21-59.27$ & $41.99-87.42$ & $22.21-59.27$ \\
$\mathrm{FN} \%$ & 21.15 & 8.89 & 60 \\
$95 \% \mathrm{Cl}(\mathrm{FN})$ & $10.05-32.25$ & $0.57-17.20$ & $29.64-90.36$ \\
$\mathrm{PPV} \%$ & 78.85 & 78.85 & 26.67 \\
$95 \% \mathrm{Cl}(\mathrm{PPV})$ & $67.75-89.95$ & $67.75-89.95$ & $4.29-49.05$ \\
$\mathrm{NPV} \%$ & 59.26 & 60 & 72.73 \\
$95 \% \mathrm{Cl}(\mathrm{NPV})$ & $40.73-77.79$ & $29.64-90.36$ & $54.12-91.34$ \\
OR & 5.42 & 5.59 & 0.97 \\
$95 \% \mathrm{Cl}(\mathrm{OR})$ & $1.95-14.97$ & $1.34-23.34$ & $0.22-4.26$ \\
\hline
\end{tabular}

Note:-Sen indicates sensitivity; Spe, specificity; FP, false-positive; FN, false-negative; NPV, negative predictive value; PPV, positive predictive value.

a The columns refer to each comparison between the groups in the independent samples.

color Doppler sonography in the same dynamic (ie, sitting and supine positions) examinations. On the other hand, MR venography, phase-contrast MR imaging, perfusion MR imaging, and the so-called criterion standard, venography, cannot provide jointly the qualitative and quantitative features of the venous system or CVF.

CVF has been demonstrated to change depending on different positions. ${ }^{19,32}$ The major drainage in the supine position is usually by the internal jugular veins. Postural dependency of the CVF has been demonstrated in healthy subjects by quantitative color Doppler sonography. ${ }^{17,18}$ A previous article ${ }^{17}$ showed that the presence of negative $\Delta \mathrm{CVF}$ is statistically correlated with a pathologic condition. The measurement of $\triangle \mathrm{CVF}$ demonstrated a statistical difference between patients with MS and the HC group in the supine and sitting positions. The higher the blood volume difference is between the supine and sitting positions, the higher is the adaptability of the cerebral venous system. Therefore, healthy subjects with normal supine/orthostatic responses show a high blood volume difference. In patients with MS, this venous response is statistically reduced. The previous study was based only on 2 groups of subjects (ie, MS and HC) and was not blinded.

The analysis of the results reported here suggests the following considerations:

1) The $\Delta$ CVF cutoff value of 503.24 correctly diagnosed a larger number of patients with MS, despite the detriment of an increased number of false-positives.

2) $\Delta \mathrm{CVF} \leq 503.24$ allowed differentiating MS versus $\mathrm{HC}$ and MS versus OND.

The distributions of the variable $\triangle \mathrm{CVF}$ in the HC and OND groups largely overlapped. On the other hand, the difference between the $\triangle \mathrm{CVF}$ in the OND and MS groups is statistically significant.

These data demonstrate that in some patients with MS, there is a hemodynamic alteration resulting in a reduced cerebral venous outflow in the supine position, most likely from decreased vertebral and internal jugular vein outflow. ${ }^{17}$ The present study also confirmed that the reduced outflow was not correlated with stenosis and dynamic or morphologic leaflet alterations. Furthermore, the reduced CVF has been demonstrated in very young patients without any venous malformations. A possible explanation is that the active tension imparted by the smooth muscle layer of the veins is not sufficient to overcome transmural pressure. In the supine position, a lower venous wall tone is not sufficient to hold venous outflow, while in the sitting position, the physiologic collapse of the main drainage veins (ie, internal jugular veins) always overcomes the low vein wall tone. This deregulation might be due to a reduced responsiveness of the vessel wall because homeostasis might be lost in changing positions. Previous observations suggested that the autonomic nervous system may be intimately linked with the disordered immune regulation in MS. Vasoactive factors such as endothelin-1 and nitric oxide may play a role in the responsiveness of the vessel wall. ${ }^{33-38}$

Another possible explanation is that this abnormal venous response is secondary to white matter hypoperfusion, and its possible mechanisms and pathophysiology were reported by De Keyser et $\mathrm{al}^{39}$

\section{CONCLUSIONS}

The present study showed that a cutoff of abnormal CVF could discriminate patients with MS from those with OND and HC.

\section{ACKNOWLEDGMENTS}

We thank Roberta Benvenuti, Roberta Baldi, Tiziana Caselli, Massimiliano Senesi, and Elena Spolaor for their technical assistance.

\section{REFERENCES}

1. Theron J, Moret J. Spinal Phlebography. Lumbar and Cervical Techniques. Berlin: Springer-Verlag; 1978

2. Zivadinov R, Karmon Y, Dolic K, et al. Multimodal noninvasive and invasive imaging of extracranial venous abnormalities indicative of CCSVI: results of the PREMiSe pilot study. BMC Neurol 2013;13:151

3. Macgowan CK, Chan KY, Laughlin S, et al. Cerebral arterial and venous blood flow in adolescent multiple sclerosis patients and agematched controls using phase contrast MRI. J Magn Reson Imaging 2013 Sep 30. [Epub ahead of print]

4. Debernard L, Melzer TR, Van Stockum S, et al. Reduced grey matter perfusion without volume loss in early relapsing-remitting multiple sclerosis. J Neurol Neurosurg Psychiatry 2014;85:544-51

5. Varga AW, Johnson G, Babb JS, et al. White matter hemodynamic abnormalities precede sub-cortical gray matter changes in multiple sclerosis. J Neurol Sci 2009;282:28-33

6. Zamboni P, Consorti G, Galeotti R, et al. Venous collateral circulation of the extracranial cerebrospinal outflow routes. Curr Neurovasc Res 2009;6:204-12

7. Menegatti E, Zamboni P. Doppler haemodynamics of cerebral venous return. Curr Neurovasc Res 2008;5:260-65

8. Zamboni P, Galeotti R, Menegatti E, et al. Chronic cerebrospinal venous insufficiency in patients with multiple sclerosis. J Neurol Neurosurg Psychiatry 2009;80:392-99

9. Hojnacki D, Zamboni P, Lopez-Soriano A, el al. Use of neck magnetic resonance venography, Doppler sonography and selective venography for diagnosis of chronic cerebrospinal venous insufficiency: a pilot study in multiple sclerosis patients and healthy controls. Int Angiol 2010;29:127-39

10. Zamboni P, Galeotti R, Menegatti E, et al. A prospective open-label study of endovascular treatment of chronic cerebrospinal venous insufficiency. J Vasc Surg 2009:50:1348-58. Erratum in J Vasc Surg 2009;51:1079

11. Mayer CA, Pfeilschifter W, Lorenz MW, et al. The perfect crime? 
CCSVI not leaving a trace in MS. J Neurol Neurosurg Psychiatry 2011;82:436-40

12. Baracchini C, Perini P, Calabrese M, et al. No evidence of chronic cerebrospinal venous insufficiency at multiple sclerosis onset. Ann Neurol 2011;69:90-99

13. Doepp F, Paul F, Valdueza JM, et al. No cerebrocervical venous congestion in patients with multiple sclerosis. Ann Neurol 2010;68:173-83

14. Khan O, Filippi M, Freedman MS, et al. Chronic cerebrospinal venous insufficiency and multiple sclerosis. Ann Neurol 2010;67:286-90

15. D'haeseleer M, Cambron M, Vanopdenbosch L, et al. Vascular aspects of multiple sclerosis. Lancet Neurol 2011;10:657-66

16. Mancini M, Morra VB, Di Donato O, et al. Multiple sclerosis: cerebral circulation time. Radiology 2012;262:947-55

17. Monti L, Menci E, Ulivelli M, et al. Quantitative colour Doppler sonography evaluation of cerebral venous outflow: a comparative study between patients with multiple sclerosis and controls. PLoS One 2011;6:e25012

18. Valdueza JM, Schmierer K, Mehraein S, et al. Assessment of normal flow velocity in basal cerebral veins: a transcranial Doppler ultrasound study. Stroke 1996;27:1221-25

19. Valdueza JM, von Münster T, Hoffman O, et al. Postural dependency of the cerebral venous outflow. Lancet 2000;355:200-01

20. Gisolf J, van Lieshout JJ, van Heusden K, et al. Human cerebral venous outflow pathway depends on posture and central venous pressure. J Physiol 2004;560:317-27

21. Wang DC, Klatzky R, Wu B, et al. Fully automated common carotid artery and internal jugular vein identification and tracking using B-mode ultrasound. IEEE Trans Biomed Eng 2009;56:1691-99

22. Dörfler P, Puls I, Schliesser M, et al. Measurement of cerebral blood flow volume in extra cranial sonography. J Cereb Blood Flow Metab 2000;20:269-71

23. Doepp F, Schreiber SJ, von Münster T, et al. How does the blood leave the brain? A systematic ultrasound analysis of cerebral venous drainage patterns. Neuroradiology 2004;46:565-70

24. Schäfer $\mathrm{H}$. Constructing a cut off point for a quantitative diagnostic test. Stat Med 1989;8:1381-91

25. Metz CE. Receiver operating characteristic analysis: a tool for the quantitative evaluation of observer performance and imaging systems. J Am Coll Radiol 2006;3:413-22

26. Hanley JA, McNeil BJ. The meaning and use of the area under a receiver operating characteristic (ROC) curve. Radiology 1982;143:29-36

27. Swets JA. Measuring the accuracy of diagnostic systems. Science 1988;240:1285-93

28. Lepori D, Capasso P, Fournier D, et al. High resolution ultrasound evaluation of internal jugular venous valves. Eur Radiol 1999;9:1222-26

29. Schreiber SJ, Lurtzing F, Gotze R, et al. Extrajugular pathways of human cerebral venous blood drainage assessed by duplex ultrasound. J Appl Physiol 2003;94:1802-05

30. Chou $\mathrm{CH}$, Chao $\mathrm{AC}, \mathrm{Hu} \mathrm{HH}$. Ultrasonographic evaluation of vertebral venous valves. AJNR Am J Neuroradiol 2002;23:1418-20

31. Renard M, Masson JP, Lardé D. Anatomo-radiological study of the spinal venous system of the lumbo-sacral junction [in French]. Bull Assoc Anat (Nancy)1975;59:725-35

32. Alperin N, Hushek SG, Lee SH, et al. MRI study of cerebral blood flow and CSF flow dynamics in an upright posture: the effect of posture on the intracranial compliance and pressure. Acta Neurochir Suppl 2005;95:177-81

33. Pache M, Kaiser HJ, Akhalbedashvili N, et al. Extraocular blood flow and endothelin-1 plasma levels in patients with multiple sclerosis. Eur Neurol 2003;49:164-68

34. Flachenecker P, Reiners K, Krauser M, et al. Cardiovascular autonomic dysfunction in multiple sclerosis: correlation with orthostatic intolerance. J Neurol 1999;246:578-86

35. Olufsen MS, Ottesen JT, Tran HT, et al. Blood pressure and blood flow variation during postural change from sitting to standing: model development and validation. J Appl Physiol 2005;99:1523-37

36. Purkayastha $S$, Raven PB. The functional role of the alpha-1 adrenergic receptors in cerebral blood flow regulation. Indian J Pharmacol 2011;43:502-06

37. Sternberg Z. Autonomic dysfunction: a unifying multiple sclerosis theory, linking chronic cerebrospinal venous insufficiency, vitamin D(3), and Epstein-Barr virus. Autoimmun Rev 2012; $12: 250-59$

38. Capone C, Faraco G, Coleman C, et al. Endothelin 1-dependent neurovascular dysfunction in chronic intermittent hypoxia. Hypertension 2012;60:106-13

39. De Keyser J, Steen C, Mostert JP, et al. Hypoperfusion of the cerebral white matter in multiple sclerosis: possible mechanisms and pathophysiological significance. J Cereb Blood Flow Metab 2008;28:1645-51 